\title{
Towards the NNLO evolution of polarised parton distributions
}

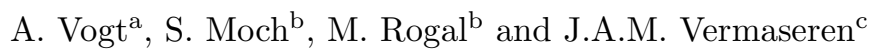

${ }^{a}$ Department of Mathematical Sciences, University of Liverpool, Liverpool L69 3BX, United Kingdom

${ }^{\mathrm{b}}$ Deutsches Elektronensynchrotron DESY, Platanenallee 6, D-15738 Zeuthen, Germany

${ }^{\mathrm{c}}$ NIKHEF, Kruislaan 409, 1098 SJ Amsterdam, The Netherlands

We report on the first calculation of the structure function $g_{1}$ in polarised deep-inelastic scattering to the third order in massless perturbative QCD. The calculation follows the dispersive approach already used for the corresponding unpolarised cases of $F_{2, L}$, but additionally involves higher tensor integrals and the Dirac matrix $\gamma_{5}$ in $D \neq 4$ dimensions. Our results confirm all known two-loop expressions including the coefficient functions of Zijlstra and van Neerven not independently verified before. At three loops we extract the helicity-difference next-to-next-to-leading order (NNLO) quark-quark and gluon-quark splitting functions $\Delta P_{\mathrm{qq}}$ and $\Delta P_{\mathrm{qg}}$. The results exhibit interesting features concerning sum rules and the momentum-fraction limits $x \rightarrow 1$ and $x \rightarrow 0$.

\section{Introduction}

Two decades after the seminal EMC measurement [1] set off the so-called spin crisis, the internal spin structure of the nucleon remains a very active field of research. By now the measurements of polarised (spin-dependent) deep-inelastic scattering (DIS) - the very process first measured by EMC - have reached a high accuracy but are still restricted to relatively small scales $Q^{2}$, see Fig. 1.

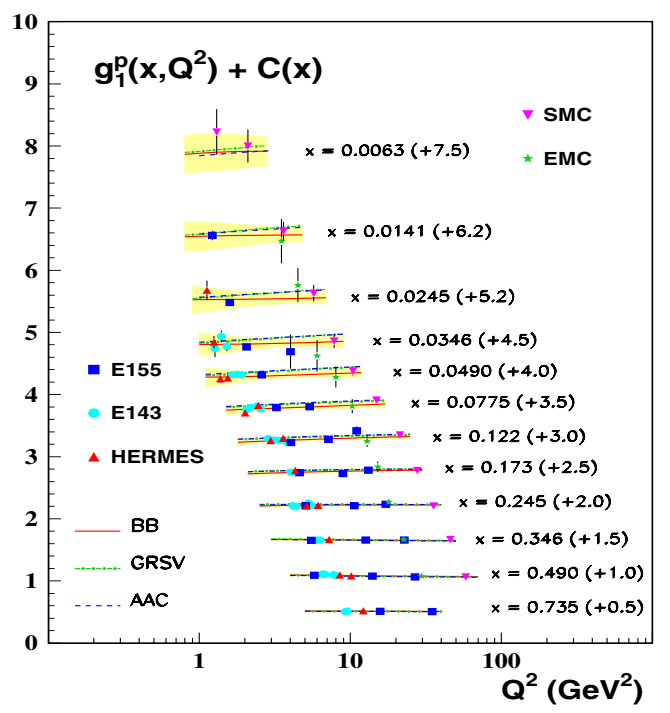

Figure 1. The end-2006 world data on the proton structure function $g_{1}$ with some NLO QCD fits [2]
Dramatic further improvements, in particular of the kinematic coverage, can be expected from the planned Electron-Ion Collider, see Ref. [3]. But, as rather low values of $Q^{2}$ imply rather large QCD corrections, even analyses of available data will profit from calculations beyond the present next-to-leading order (NLO) approximation.

\section{Polarised DIS in perturbative QCD}

The kinematics and perturbative-QCD factorization of inclusive DIS are recalled in Fig. 2.

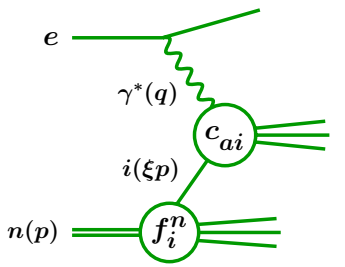

Scale, Bjorken variable

$$
\begin{aligned}
Q^{2} & =-q^{2} \\
x & =Q^{2} /(2 p \cdot q)
\end{aligned}
$$

Lowest order : $x=\xi$

Figure 2. DIS in the QCD-improved parton model

The measurements of the difference $\sigma_{\vec{e} \vec{n}}-\sigma_{\vec{e} \leftarrow}$ of the helicity-dependent cross sections yield the polarised structure function $g_{1}^{n}$ of the nucleon $n$. In perturbative QCD, neglecting contributions suppressed by $1 / Q^{2}$, this quantity is given by

$$
g_{1}^{n}\left(x, Q^{2}\right)=\int_{x}^{1} \frac{d \xi}{\xi} c_{g_{1}, k}\left(\frac{x}{\xi}, \alpha_{\mathbf{s}}\left(\mu^{2}\right), \frac{\mu^{2}}{Q^{2}}\right) \Delta f_{k}^{n}\left(\xi, \mu^{2}\right)
$$


where, as in Eq. (2) below, the sum over the parton species $k$ is understood. $c_{g_{1}, k}$ represents the corresponding coefficient functions (massfactorized partonic cross sections) for $g_{1}, \alpha_{\mathrm{s}}$ denotes the strong coupling, and $\mu=\mathcal{O}(Q)$ stands for the renormalization and mass-factorization scale. The helicity-difference parton distributions $\Delta f_{i}=f_{i \rightarrow}-f_{i \leftarrow}$ obey the evolution equations

$\frac{d}{d \ln \mu^{2}} \Delta f_{i}\left(\mu^{2}\right)=\left[\Delta P_{i k}\left(\alpha_{\mathrm{s}}\left(\mu^{2}\right)\right) \otimes \Delta f_{k}\left(\mu^{2}\right)\right]$

where $\otimes$ denotes the Mellin convolution written out in Eq. (1). The initial conditions for Eq. (2) are, of course, incalculable in perturbative QCD. Present lattice techniques can provide, at best, only a few low Mellin moments. Hence predictions for, e.g., processes at RHIC, can be obtained only via fit-analyses of reference observables such as $g_{1}$ and the universality of the parton densities. For the present state-of-the-art see, e.g., Ref. [4].

The perturbative quantities entering a general hard-scattering observable $a$ are thus the splitting functions $P$ and the coefficient functions $c_{a}$,

$$
\begin{aligned}
P & =\alpha_{\mathrm{s}} P^{(0)}+\alpha_{\mathrm{s}}^{2} P^{(1)}+\alpha_{\mathrm{s}}^{3} P^{(2)}+\ldots \\
c_{a} & =\underbrace{\alpha_{\mathrm{s}}^{n_{a}}\left[c_{a}^{(0)}+\alpha_{\mathrm{s}} c_{a}^{(1)}\right.}_{\text {NLO }}+\alpha_{\mathrm{s}}^{2} c_{a}^{(2)}+\ldots] .
\end{aligned}
$$

Being the first serious approximation, the NLO cannot provide a sound estimate of the error from the truncation of the perturbation series. The NNLO requires the two-loop coefficient functions for all observables under consideration and the universal three-loop splitting functions. The latter quantities $\Delta P_{i k}^{(2)}$ for the polarised evolution (2) are the main subject of the present research.

\section{Previous second-order calculations}

Before we turn to our new third-order calculations, it is useful to briefly recall the previous two-loop results for the coefficient functions for $g_{1}$ and the splitting functions $\Delta P_{i k}$. All those calculations have been carried out in dimensional regularization. Hence the helicity-difference projection of initial quarks introduces the issue of $\gamma_{5}$ in $D \neq 4$ dimensions into the problem.
The perturbative corrections for the polarised structure function $g_{1}$ to order $\alpha_{\mathrm{s}}^{2}$ have been calculated 15 years ago by Zijlstra and van Neerven [5]. This $x$-space calculation, carried out using the socalled Larin scheme [6] for $\gamma_{5}$ (see below), yielded the NNLO coefficient functions $c_{g_{1}, \mathrm{q} / \mathrm{g}}^{(2)}$ and well as two of the four polarised NLO splitting functions: $\Delta P_{\mathrm{qq}}^{(1)}$ and $\Delta P_{\mathrm{qg}}^{(1)}$.

The complete set of NLO splitting functions $\Delta P_{i j}^{(1)}$ was first computed two years later by Mertig and van Neerven using the operator-product expansion [7]. Here $\gamma_{5}$ was treated using the reading-point method of Ref. [8]. The results were independently verified already a few months later by Vogelsang [9] in an axial-gauge calculation. This calculation was performed using the original HVBM prescription for $\gamma_{5}$ [10] but included checks with the other schemes mentioned above.

All three theoretically (but not computationally) identical prescriptions for $\gamma_{5}$ require an additional renormalization or factorization of the loop results in Refs. [5,7,9]. The complete set of the corresponding expressions for $g_{1}$ to NNLO has been derived in Ref. [11] ten years ago. We will return to this issue below.

\section{The three-loop computation via forward Compton amplitudes}

The present calculation follows the approach used before in our computation of the unpolarised splitting functions [12] (see also Ref. [13]) and the coefficient functions for $F_{2, L}[14]$ to order $\alpha_{\mathrm{s}}^{3}$. Thus we employ the optical theorem

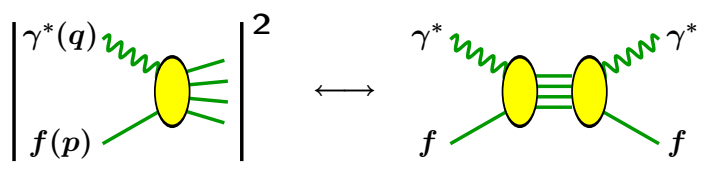

to transform the $\gamma^{*} f$ total cross sections into forward Compton amplitudes. Due to a dispersion relation in the partonic Bjorken variable $x$, the coefficient of $(2 p \cdot q)^{N}$ provides the $N$-th moment

$$
\hat{F}_{a}^{N}=\int_{0}^{1} d x x^{N-1} \hat{F}_{a}(x)
$$

of the partonic structure function $\hat{F}_{a}$ selected by a suitable projection of the $\gamma^{*}$-amputated graphs. 
In particular, the projection of the partonic tensor $\widehat{W}^{\mu \nu}$ on $\hat{g}_{1}$ in $D$ dimensions reads [5]

$$
\hat{g}_{1}=2[(D-2)(D-3)(p \cdot q)]^{-1} \varepsilon_{\mu \nu p q} \widehat{W}^{\mu \nu}
$$

using the SchOONSCHIP notation for contracted indices. The $\mathrm{N}^{l-1} \mathrm{LO}$ splitting functions $\Delta P_{\mathrm{qf}}^{(l-1)}$ and the $\mathrm{N}^{l} \mathrm{LO}$ coefficient functions $c_{g_{1}, \mathrm{f}}^{(l)}$ can be extracted from the $1 / \varepsilon$ poles $(D=4-2 \varepsilon)$ and $\varepsilon^{0}$ parts, respectively, of the corresponding $l$-loop results for $\gamma^{*} f \rightarrow \gamma^{*} f$, cf. Refs [12-14].

\section{Treatment of the integrals}

In order to illustrate the computation of integrals required to evaluate the forward Compton amplitudes, let us consider one of the hundreds of $\gamma^{*} g$ three-loop diagrams shown here together with a pictorial representation of its momentum flow:

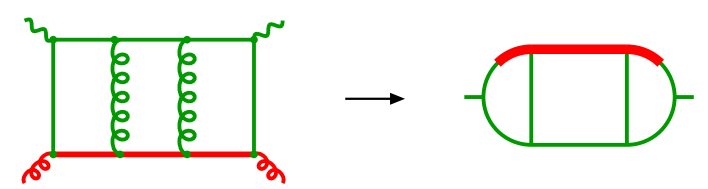

For the latter one disregards the external parton lines and draws the remaining self-energy type diagram, the topology of which is denoted following the notation of Refs. [15]. Our example is a ladder (LA) diagram. The (partly additional) denominators carrying the parton momentum $p$ are then indicated by the fat (in the coloured version: red) lines. Here $p$ runs, after turning the diagram upside-down, through the lines 1,2 and 3. Thus the example is assigned the subtopology $\mathrm{LA}_{13}$.

According to our above discussion, we need analytic expressions for the (dimensionless) coefficients $I(N)$ of $(2 p \cdot q)^{N} / Q^{2 \alpha}$. One might try to obtain $I(N)$ by brute force, Taylor-expanding the denominators with $p$ and working out the sums. It turned out that such a strategy, in general, does not work. Instead, we employed identities based on integration by parts, scaling arguments and form-factor decompositions (see Sect. 2 of Ref. $\left.[12]_{1}\right)$ to successively simplify the integrals.

The $\mathrm{LA}_{13}$ integrals, e.g., can be simplified by applying $p^{\mu} \partial / \partial q^{\mu}$ both inside and outside the integral. For the scalar integral with unit denominators this yields

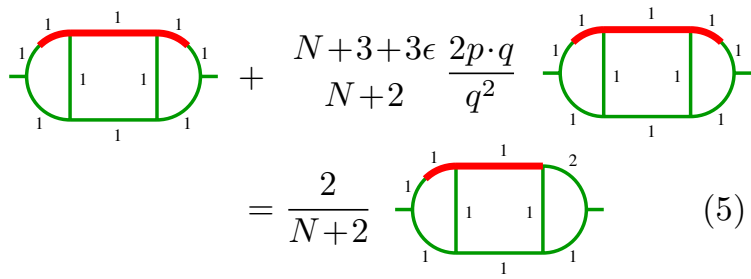

Here the $\mathrm{LA}_{13}$ integral occurs twice, once with a prefactor $2 p \cdot q$. Hence Eq. (5) represents a difference equation (here of order $n=1$ ) expressing its coefficient $I(N)$ in terms of that of a $\mathrm{LA}_{12}$ integral with an enhanced denominator in the 3 -line,

$$
a_{0}(N) I(N)-\ldots-a_{n}(N) I(N-n)=G(N) .
$$

First-order recursion relations like Eq. (5) can be reduced to a sum. Higher-order recursions (we needed equations up to $n=4$ ) can be solved by inserting a suitable ansatz into Eq. (6). Both procedures exploit the fact that all integrals required for the computation of the splitting functions can be expressed in terms of harmonic sums [16].

Despite being uncharacteristically simple in both derivation and size, Eq. (5) illustrates the strict hierarchy of subtopologies in our procedure. Our $\mathrm{LA}_{13}$ example can be evaluated only once the $\mathrm{LA}_{12}$ integral in Eq. (5) is known. This integral requires the so-called basic building blocks (with only one $p$-dependent denominator) $\mathrm{LA}_{11}$ and $\mathrm{LA}_{22}$ together with other integrals of simpler topologies where a non- $p$ denominator has been removed. Also those integrals need to be evaluated in terms of yet simpler cases, and so on.

During the first half of this decade, reduction chains for all subtopologies were constructed and coded in Form [17]. This and the computation of all integrals (and their much more numerous sub-integrals) entering the diagrams for the unpolarized cases [12-14] took years of both human and computing resources. It would not have been possible to get through without extensive tabulation of intermediate results. By 2005, a database had been accumulated of more than 100000 integrals requiring about 3.5 GBytes of disk space.

However, as we will see below, this database is still not large enough to cover all integrals required for the three-loop calculation of the spindependent structure function $g_{1}$. 


\section{Numerators for a non-planar diagram}

One of the more difficult three-loop diagrams for the $\gamma^{*} q$ Compton amplitudes is shown below in fully amputated form:

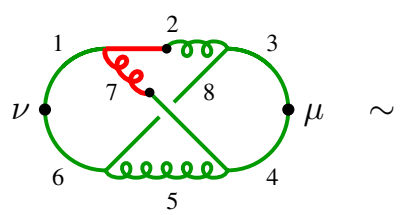

$\frac{\gamma\left(i_{1}, p_{7}, i_{2}, p_{4}, \mu, p_{3}, i_{3}, p_{8}, i_{2}, p_{6}, \nu, p_{1}, i_{1}, p+p_{2}, i_{3}\right)}{p_{1}^{2} \ldots p_{8}^{2}\left(p+p_{2}\right)^{2}\left(p+p_{7}\right)^{2}}$

Here the numerator has been written in the notation used by FORM [17], and the numbers in the graph correspond to the line numbering convention in Mincer [15]. Thus this diagram, with the quark entering in line 2 and leaving in line 7 , is of the subtopology $\mathrm{NO}_{27}$.

In order to determine the unpolarised splitting function, it is sufficient to evaluate $(7) \cdot \gamma(p) g_{\mu \nu}$. The resulting trace leads to numerators

$$
\left(p_{2} \cdot p\right)^{k_{2}}\left(p_{3} \cdot p\right)^{k_{3}}\left(p_{2} \cdot q\right)^{k_{9}} \text { with } k_{2}+k_{3}+k_{9} \leq 3
$$

and the denominator $1 / p_{2}^{2}$ cancels in all terms, which already reduces the number of required reduction steps by one.

The disentangling of the contributions to the coefficient functions for $F_{2}$ and $F_{L}$ required the additional calculation of $(7) \cdot \gamma(p) p_{\mu} p_{\nu} /(p \cdot q)^{2}$ which includes higher numerators,

$\left(p_{2} \cdot p\right)^{k_{2}}\left(p_{3} \cdot p\right)^{k_{3}}\left(p_{2} \cdot q\right)^{k_{9}}$ with $k_{2}+k_{3}+k_{9} \leq 4$.

Again no denominator $1 / p_{2}^{2}$ remains in any term.

The determination of the spin splitting function and the coefficient function for $g_{1}$ requires the evaluation of $(7) \cdot \gamma(p, 5) \varepsilon_{\mu \nu p q} /(p \cdot q)$ with the $\gamma$-term arising from the quark helicity-difference and the $\varepsilon$-tensor from the $g_{1}$ projection in Eq. (4). Now one encounters yet higher numerators

$$
\left(p_{2} \cdot p\right)^{k_{2}}\left(p_{3} \cdot p\right)^{k_{3}}\left(p_{2} \cdot q\right)^{k_{9}} \text { with } k_{2}+k_{3}+k_{9} \leq 5
$$

and the denominators $1 / p_{2}^{2}$ remain in terms up to the highest numerators. Thus, compared to the previous calculations of $F_{2}$ and $F_{L}$ [12-14], two additional reduction steps (often leading to additional denominator enhancements, recall Eq. (5)) are required in the polarised case.
The situation is similar in other difficult subtopologies including $\mathrm{NO}_{12}, \mathrm{BE}_{68}$ and $\mathrm{LA}_{17}$ (some of which, unfortunately, are far from being fully automated), leading to a large number of new (sub-)integrals beyond the 2005 database.

Using codes and experience from the previous unpolarized calculations, we were able to compute the required integrals within a couple of months. This would have been impossible without the possibility of fixed- $N$ checks at all intermediate stages facilitated by the MINCER program [15].

\section{Treatment of $\gamma_{5}$ and two-loop checks}

We employ the Larin prescription for the quark helicity-difference projector, i.e., we use [6]

$$
\not p \gamma_{5, L}=\frac{i}{6} \varepsilon_{p \mu \nu \rho} \gamma_{\mu} \gamma_{\nu} \gamma_{\rho} .
$$

Hence, after coupling-constant renormalization and mass-factorization, we need to perform a scheme transformation $Z$ which nullifies the first moment of the non-singlet (ns) quark-quark splitting function in accordance with the conservation of the non-singlet axial vector current $[7,9]$,

$$
\begin{aligned}
g_{1} & =c_{g_{1}, L} \Delta f_{L} \\
& =c_{g_{1}, L} Z^{-1} Z \Delta f_{L}=c_{g_{1}, \overline{\mathrm{MS}}} \Delta f_{\overline{\mathrm{MS}}} .
\end{aligned}
$$

The general form of the corresponding transformation of the splitting functions to NNLO in the flavour-singlet case, where Eq. (9) has to be read as a matrix equation, can be found, e.g., in Sect. 2 of Ref. [18]. For the special case

$$
Z_{i j}=\delta_{i j}+a_{\mathrm{s}} z_{q q}^{(1)} \delta_{q q}+a_{\mathrm{s}}^{2}\left(z_{q q, \mathrm{~ns}}^{(2)}+z_{q q, \mathrm{ps}}^{(2)}\right) \delta_{q q}
$$

the ( $x$-space) transformations of the NLO and NNLO parton-quark splitting functions read

$$
\begin{aligned}
& \delta\left[\Delta P_{q q}^{(1)}\right]=-\beta_{0} z_{q q}^{(1)}, \\
& \delta\left[\Delta P_{q g}^{(1)}\right]=z_{q q}^{(1)} \otimes \Delta P_{q g}^{(0)}
\end{aligned}
$$

and

$$
\begin{aligned}
& \delta\left[\Delta P_{q q}^{(2)}\right]=\beta_{0}\left[\left(z_{q q}^{(1)}\right)^{\otimes 2}-2 z_{q q}^{(2)}\right]-\beta_{1} z_{q q}^{(1)}, \\
& \delta\left[\Delta P_{q g}^{(2)}\right]=z_{q q}^{(2)} \otimes \Delta P_{q g}^{(0)}+z_{q q}^{(1)} \otimes \Delta P_{q g, L}^{(1)} .
\end{aligned}
$$

The non-singlet entries in Eq. (10) can be inferred from the fact that the $\overline{\mathrm{MS}}$ coefficient functions for $g_{1}$ and the structure function $F_{3}^{\nu+\bar{\nu}}$ in 
neutrino-nucleon DIS are identical to two loops. The functions thus determined agree with the results of the direct calculation in Ref. [11]. At the moment the latter article is the only source for the NNLO pure-singlet transformation term $z_{q q, \mathrm{ps}}^{(2)}$.

After transforming to the $x$-space expressions in terms of harmonic polylogarithms [19] and performing the transformation (9)-(11), our twoloop expressions fully agree with the previous results for $\Delta P_{\mathrm{qf}}^{(1)}$ and $c_{g_{1}, \mathrm{f}}^{(2)}$ discussed in Section 3. Incidentally, we note that the change of $c_{g_{1}}^{(2)}$ ps in the second erratum of [5] is exactly the scheme transformation induced by $z_{q q, \mathrm{ps}}^{(2)}$ of Ref. [11].

\section{Checks and features of the 3-loop results}

A strong check of the third-order computations is provided by the $\varepsilon^{-3}$ and $\varepsilon^{-2}$ pole terms of the unfactorized partonic structure functions. These terms have to be of a specific form in terms of the lower-order splitting functions and coefficient functions, including the NLO quantities $\Delta P_{\mathrm{gf}}^{(1)}$ of Refs. $[7,9]$ inaccessible to a two-loop calculation of photon-exchange DIS. Our results pass this test.

We find that $\Delta P_{\mathrm{ns}}^{(2)}$ in Eq. (3) is equal to the unpolarized non-singlet splitting function $P_{\mathrm{ns}}^{(2)-}$ after the scheme transformation (12) adjusting $c_{g_{1}, \text { ns }}^{(2)}$ to the $(\nu+\bar{\nu})$ coefficient function $c_{F_{3}}^{(2)}[20]$. This guarantees that the first moment of $\Delta P_{\mathrm{ns}}^{(2)}$ vanishes as required by the conservation of the non-singlet axial vector current.

Also the first moment of the NNLO gluonquark splitting function vanishes

$$
\Delta P_{\mathrm{qg}}^{(2)}(N=1)=0
$$

in agreement with the 1990 result of Altarelli and Lampe [21]. For the pure singlet the relation

$$
\Delta P_{\mathrm{ps}}^{(n)}(N=1)=-2 n_{f} \Delta P_{\mathrm{gq}}^{(n-1)}(N=1)
$$

also holds at $\operatorname{NNLO}(n=2)$. Eqs. (13) and (14) suggest the possibility of a higher-order generalisation which would bring us closer to a complete NNNLO description of $g_{1}$ at $N=1$.

Turning to large $x$, we note that the helicity flip is suppressed by two powers of $(1-x)$ as $x \rightarrow 1$,

$$
P_{\mathrm{qg}}^{(2)}(x)-\Delta P_{\mathrm{qg}}^{(2)}(x) \sim(1-x)^{2} \cdot P_{\mathrm{qg}}^{(2)}(x)
$$

(the corresponding relation for $\Delta P_{\mathrm{qq}}^{(2)}$ is obvious as only the suppressed pure-singlet parts differ), where $P_{\mathrm{qg}}$ denotes the unpolarized (flip + nonflip) splitting function. This is in line with the pattern advocated by Brodsky et al. [22] for the perturbative and non-perturbative contributions to the helicity-dependent parton distributions. It is interesting to note that the same suppression holds for 7 of the $8 \mathrm{LO}$ and NLO splitting functions $\Delta P_{i j}^{(0,1)}$, the curious exception being $\Delta P_{\mathrm{gq}}^{(1)}$ (we are not aware of any earlier discussion of this). A simple scheme transformation $\left(z_{g q}^{(1)} \propto \Delta P_{\mathrm{gq}}^{(0)}\right)$ exists which would 'repair' this exception.

Since it is identical to the unpolarized quantity $P_{\mathrm{ns}}^{(2)-}$, the leading small- $x$ logarithm of $\Delta P_{\mathrm{ns}}^{(2)}$ agrees with the prediction of the small- $x$ resummation [23]. The situation is less straightforward in the singlet sector where we find

$$
\begin{aligned}
\left.\Delta P_{\mathrm{ps}}^{(2)}\right|_{\ln ^{4} x} & =-C_{F} n_{f}\left(2 C_{A}+\frac{8}{3} C_{F}\right) \\
\left.\Delta P_{\mathrm{qg}}^{(2)}\right|_{\ln ^{4} x} & =-5 C_{A}^{2} n_{f}-\frac{4}{3} C_{F} n_{f}\left(C_{A}-n_{f}\right)
\end{aligned}
$$

$\left(C_{A}\right.$ and $C_{F}$ are the usual group factors of QCD). The first of Eqs. (16) agrees with the 1996 prediction of Blümlein and Vogt [24] based on the singlet resummation by Bartels et al. [25]. However, the corresponding coefficients of $C_{A} C_{F} n_{f}$ and $C_{F}^{2} n_{f}$ of $\Delta P_{\mathrm{qg}}^{(2)}$ are not the same. Agreement is restored in the supersymmetric limit.

We have to leave the clarification of this point to further studies. However, we should note here that, unlike in the non-singlet case, the leading small- $x \operatorname{logs}$ of the singlet splitting functions are not scheme invariant. This can be seen from the second lines of Eqs.(11) and (12): for $z_{q q}^{n} \propto$ $c_{g_{1}, \mathrm{q}}^{(n)}$, for example, $\delta\left[\Delta P_{\mathrm{qg}}^{(n)}\right]$ will include leading$\log$ contributions (recall that $\ln ^{k} x \sim 1 / N^{k+1}$ in $N$-space). Hence one cannot conclude now that the above difference constitutes an inconsistency.

A final remark is in order on the $\gamma_{5}$-induced transformation to the $\overline{\mathrm{MS}}$ factorization scheme. An additional term $z_{g q}^{(2)}$ can be expected from the use of, e.g., the Larin scheme in a calculation of an observable suitable to access $\Delta P_{\mathrm{gq}}^{(2)}$ (see below). This presently unknown function would add an additional term $-z_{g q}^{(2)} \otimes \Delta P_{\mathrm{qg}}^{(0)}$ to the first line of Eq. (12) without affecting the result for $\Delta P_{\mathrm{qg}}^{(2)}$. 
Fig. 3 finally illustrates the perturbative expansion of the helicity-difference gluon-quark splitting function to the next-to-next-to-leading order. Here we have employed the code of Ref. [26] for the numerical evaluation of the harmonic polylogarithms [19] up to weight four.
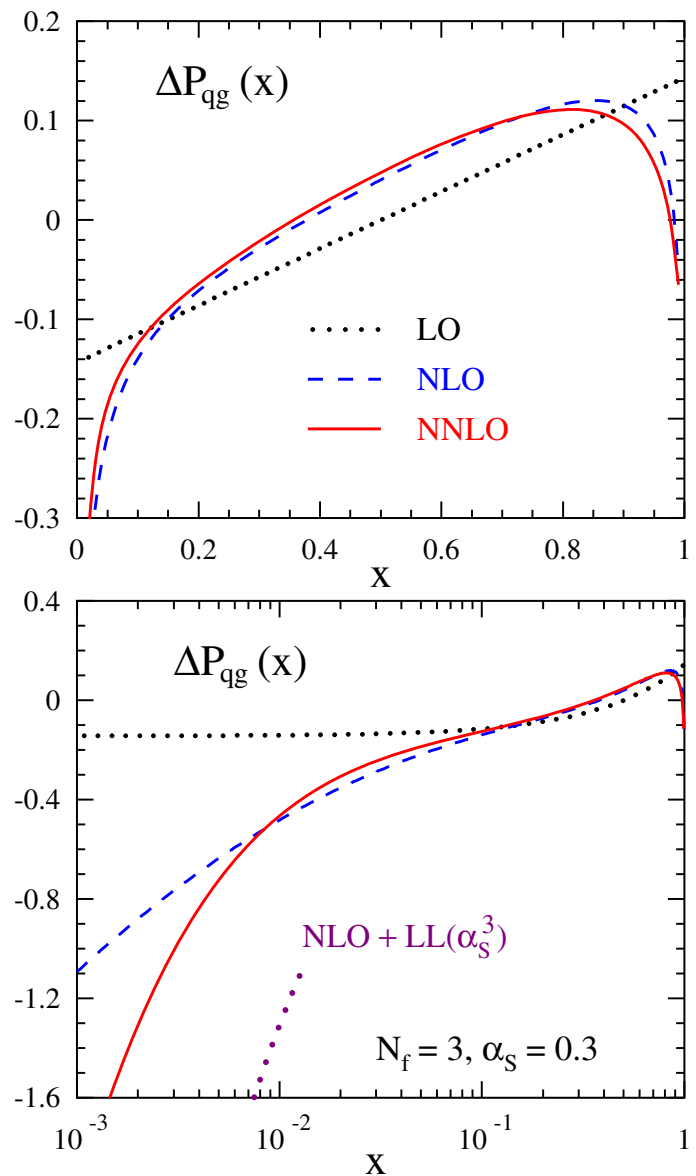

Figure 3. The LO, NLO, and NNLO approximations for the splitting function $\Delta P_{\mathrm{qg}}$ at values of $\alpha_{\mathrm{s}}$ and the number $n_{f}$ of light flavours relevant to present data on the spin structure function $g_{1}$.

The third-order corrections are $15 \%$ or less over the wide region $0.005 \leq x \leq 0.9$ even for this relatively large value of $\alpha_{\mathrm{s}}$. The figure also shows that, as for all other three-loop quantities we have calculated [12-14] (see also Refs. [27] for the timelike case), the leading small- $x$ logarithm does not provide a useful approximation to the complete function at any relevant values of $x$.

\section{Summary and Outlook}

We have calculated the three-loop QCD corrections to spin-dependent electromagnetic deepinelastic scattering and extracted the upper row $\Delta P_{\mathrm{qf}}^{(2)}(x), f=q, g$, of the matrix of the thirdorder (NNLO) spin splitting functions.

Due to the presence of difficult tensor integrals beyond those encountered before [12-14], the computation of the corresponding diagrams provided yet another example of the 'no free lunch' theorem. The $D \neq 4$ continuation of $\gamma_{5}$ has been dealt with using the Larin prescription [6] and, as in previous two-loop calculations $[7,9]$, a final scheme transformation to $\overline{\mathrm{MS}}$ factorization. The result for $\Delta P_{\mathrm{qg}}^{(2)}$ is final also in this respect, while the pure-singlet quark splitting function $\Delta P_{\mathrm{ps}}^{(2)}$ may require an additional transformation.

Our calculation also includes the coefficient functions for the spin structure function $g_{1}$ up to the NNNLO (again in $\overline{\mathrm{MS}}$ up to a scheme transformation in the pure-singlet sector). At two loops we confirm the previous results for the NNLO coefficient functions $c_{g_{1}, \mathrm{f}}^{(2)}$ of Ref. [5] which were not independently checked before. Returning to the NNLO splitting functions, we note that our results exhibit interesting features at the moment $N=1$ and the momentum-fraction limits $x \rightarrow 1$ and $x \rightarrow 0$ which merit further studies.

We have started work on the lower row $\Delta P_{\mathrm{gf}}^{(2)}$, $f=q, g$ of the splitting-function matrix. Our method is a generalisation of the scalar-exchange approach used in the unpolarized case $[12,13]$, i.e., we now consider DIS including also the exchange of a pseudoscalar (Higgs in the heavy-top limit) $\chi$ coupling to $\widetilde{G}_{\mu \nu}^{a} G_{a}^{\mu \nu}$. This adds an operator mixing to the problem [28]. Moreover, there are many more diagrams in this sector, e.g., a preliminary (non-optimised) database has about $30000 \mathrm{~g} \phi g \chi$ diagrams where $\phi$ is the scalar Higgs used before.

Despite the expected impact of improved tools such a the new multi-threaded version of FORM [29], it will thus take some time before all NNLO spin splitting functions are known. In the meantime, the full $N$ - and $x$-space results for $\Delta P_{\mathrm{qf}}^{(2)}$ (including FORM and ForTRAN routines) will be presented in a forthcoming journal publication. 


\section{Acknowledgements}

We would like to thank W. Vogelsang for useful discussions. The work of A.V. has been supported in part by the UK Science \& Technology Facilities Council under grant number PP/E007414/1. S.M. and M.R. acknowledge support by the Helmholtz Gemeinschaft under contract VH-NG-105 and by the Deutsche Forschungsgemeinschaft in Sonderforschungsbereich/Transregio 9. The work of J.V. has been part of the research program of the Dutch Foundation for Fundamental Research of Matter (FOM).

\section{REFERENCES}

1. J. Ashman et al. [European Muon Collab.], Phys. Lett. B206 (1988) 364

2. H. Böttcher, http://www-hermes.desy.de/ notes/pub/trans-public-subject.html\#G1.

3. C. Aidala et al. [EIC Working Group], April 2007 White Paper, at http://web.mit. edu/eicc/Documentation.html

4. D. de Florian, R. Sassot, M. Stratmann and W. Vogelsang, arXiv:0804.0422 [hep-ph]

5. E.B. Zijlstra and W.L. van Neerven, Nucl. Phys. B417 (1994) 61 [Errata: ibid. B426 (1994) 245; B773 (2007) 105]

6. S.A. Larin and J.A M. Vermaseren, Phys. Lett. B259 (1991) 345; S.A. Larin, Phys. Lett. B303 (1993) 113, hep-ph/9302240

7. R. Mertig and W.L. van Neerven, Z. Phys. C70 (1996) 637. Beware of the (critically different) arXiv version as of June 2008.

8. J.G. Körner, D. Kreimer and K. Schilcher, Z. Phys. C54 (1992) 503

9. W. Vogelsang, Phys. Rev. D54 (1996) 2023, hep-ph/9512218; Nucl. Phys. B475 (1996) 47, hep-ph/9603366

10. G. 't Hooft and M.J.G. Veltman, Nucl. Phys. B44 (1972) 189; P. Breitenlohner and D. Maison, Commun. Math. Phys. 52 (1977) 11

11. Y. Matiounine, J. Smith and W.L van Neerven, Phys. Rev. D58 (1998) 076002, hep-ph/ 9803439
12. S. Moch, J. Vermaseren and A. Vogt, Nucl. Phys. B688 (2004) 101, hep-ph/0403192; Nucl. Phys. B691 (2004) 129, hep-ph/0404111

13. A. Vogt, S. Moch and J. Vermaseren, Proceedings of DIS 2004, p. 231, hep-ph/0407321; Nucl. Phys. Proc. Suppl. 135 (2004) 137, hep$\mathrm{ph} / 0408075$

14. J. Vermaseren, A. Vogt and S. Moch, Phys. Lett. B606 (2005) 123, hep-ph/0411112; Nucl. Phys. B724 (2005) 3, hep-ph/0504242

15. S.G. Gorishnii, S.A. Larin, L.R. Surguladze and F.V. Tkachov, Comput. Phys. Commun. 55 (1989) 381; S.A. Larin, F.V. Tkachev and J.A.M. Vermaseren, NIKHEF-H-91-18

16. J.A.M. Vermaseren, Int. J. Mod. Phys. A14 (1999) 2037, hep-ph/9806280

17. J.A.M. Vermaseren, math-ph/0010025; Nucl. Phys. Proc. Suppl. 116 (2003) 343, hep$\mathrm{ph} / 0211297$

18. W.L. van Neerven and A. Vogt, Nucl. Phys. B588 (2000) 345, hep-ph/0006154

19. E. Remiddi and J.A.M. Vermaseren, Int. J. Mod. Phys. A15 (2000) 725, hep-ph/9905237

20. E. Zijlstra and W. van Neerven, Phys. Lett. B297 (1992) 377; S. Moch and J. Vermaseren, Nucl. Phys. B573 (2000) 853, hep-ph/9912355

21. G. Altarelli and B. Lampe, Z. Phys. C47 (1990) 315

22. S.J. Brodsky, M. Burkardt and I. Schmidt, Nucl. Phys. B441 (1995) 197, hep-ph/9401328

23. R. Kirschner and L.N. Lipatov, Nucl. Phys. B213 (1983) 122; J. Blümlein and A. Vogt, Phys. Lett. B370 (1996) 149, hep-ph/9510410

24. J. Blümlein and A. Vogt, Phys. Lett. B386 (1996) 350, hep-ph/9606254

25. J. Bartels, B.I. Ermolaev and M.G. Ryskin, Z. Phys. C72 (1996) 627, hep-ph/9603204

26. T. Gehrmann and E. Remiddi, Comp. Phys. Commun. 141 (2001) 296, hep-ph/0107173

27. S. Moch and A. Vogt, Phys. Lett. B659 (2008) 290, arXiv:0709.3899 [hep-ph]

28. K.G. Chetyrkin, B.A. Kniehl, M. Steinhauser and W.A. Bardeen, Nucl. Phys. B535 (1998) 3, hep-ph/9807241

29. M. Tentyukov and J.A.M. Vermaseren, hep$\mathrm{ph} / 0702279$ 